\title{
Unités morphopédologiques et gestion de la fertilité des sols dans le Centre-Nord du Burkina Faso par les populations locales
}

\author{
Dapola E.C. DA ${ }^{1 *}$, Hamma YACOUBA ${ }^{2}$ and Samuel YONKEU ${ }^{2}$ \\ ${ }^{1}$ Département de Géographie, Université de Ouagadougou BP 7021 Ouagadougou 03, Burkina Faso. \\ ${ }^{2}$ Institut International d'Ingénierie de l'Eau et de l'Environnement, BP 594 Ouagadougou 01, Burkina Faso. \\ *Auteur correspondant, E-mail: dapola.da@univ-ouaga.bf, Tél. : +22670283293.
}

\section{RESUME}

Le Burkina Faso, à l'image des pays sahéliens, subit les effets de la désertification. Cela est dû en grande partie à la variabilité climatique et à la dégradation du couvert végétal dont les causes sont à la fois naturelles et anthropiques. Au nombre des effets induits, la prise en compte de la baisse de la fertilité des sols semble être quelque peu négligée. Par une approche physicochimique et ethno-pédologique, cette conséquence a été abordée par la présente étude, dans le Centre-Nord du Burkina Faso. La relation entre l'organisation des unités géomorphologiques et les sols d'une part, et d'autre part entre les unités morpho pédologiques et leur perception par les populations qui les exploitent, permettent de cerner toute l'importance du modelé dans le drainage hydrique et dans la fertilité des sols. Cette connaissance constitue une véritable base pour une bonne gestion des sols; elle permet de prendre toutes les dispositions conservatoires pour l'amélioration et le maintien de la fertilité des sols en fonction des unités géomorphologiques.

(c) 2008 International Formulae Group. All rights reserved.

Mots clés : Domaine soudano-sahélien, modelé du relief, sols, gestion de la fertilité, connaissances locales.

\section{INTRODUCTION}

Le terme «dégradation des paysages» désigne les pertes de terre dans les zones arides, semi-arides et subhumides sèches par suite de divers facteurs, parmi lesquels les variations climatiques et les activités humaines (Yonkeu et al., 2003). Un sol se dégrade lorsqu'il y a perte de terre arable, d'eau et de biodiversité. Les terres arables peuvent s'éroder, se saliniser et/ou encore s'appauvrir.

Si la dégradation des terres est un phénomène qui se produit partout, elle n'est qualifiée de «désertification» que lorsqu'elle touche des terres arides. Soixante-dix pour cent des 5200 millions d'hectares de terres arides du globe utilisées pour l'agriculture sont déjà dégradés selon la FAO, citée par le PNUE (2002). La désertification est un phénomène qui menace toutes les régions sahéliennes de l'Afrique et s'étend vers les zones soudaniennes. Si la sécheresse et les activités des hommes semblent être les principales causes, les mécanismes qui aboutissent à ce phénomène sont loin d'être connus et nécessitent encore beaucoup d'investigations. La dégradation des paysages est une des principales étapes qui mènent à l'érosion et à la désertification. Aussi, la compréhension des phénomènes de dégradation des paysages reste t-elle au cœur des préoccupations de tous les pays affectés par la désertification et surtout par l'avancée notable du désert.

Le Burkina Faso est un pays enclavé du domaine sahélo-soudanien de l'Afrique de l'Ouest. La révision des perspectives mondiales des Nations Unies estimait sa population à 11,5 millions d'habitants en 2000 avec une prévision de 46,3 millions d'habitants en 2050 (Nations Unies, 1999). Le recensement de 2007 indique une population de 13.117.100 habitants (http://www.insd.bf/). On estime approximativement à $8900 \mathrm{~km}^{2}$, la 
superficie des terres arables au Burkina Faso. Ce sont majoritairement des sols de types lixisols et cambisols ferrugineux tropicaux, pauvres en matière organique (Zombré, 2006a). Néanmoins, l'économie du Burkina Faso repose sur l'agriculture et l'élevage. Le secteur agricole mobilise $90 \%$ de la population burkinabé et représente $30 \%$ du PIB national (INSD, 1996). Les techniques et les pratiques agricoles restent cependant très rudimentaires, ce qui maintient ce secteur dans une agriculture de subsistance, consommatrice d'espace et à peine suffisante pour nourrir une population à croissance rapide.

Substrat premier de l'agriculture, parmi les facteurs de production, le sol au Burkina Faso est soumis à toutes formes de pression : modes d'exploitation abusifs, croissance démographique accélérée, capital foncier en continuelle dégradation mettant en péril les systèmes de production. Ainsi la péjoration climatique, la pression démographique et les pratiques agro-pastorales ont eu pour conséquences la dégradation des sols et une baisse notable de la fertilité de ces derniers. Le Centre-Nord du Burkina Faso étant particulièrement atteint par ce phénomène, les sols y ont tendance, sous l'effet des orages, à subir des réorganisations superficielles importantes, limitant l'infiltration (Casenave et Valentin, 1989). Dans ce contexte, la gestion des sols et des eaux devient un élément essentiel de la sécurité alimentaire. En effet, la persistance de la dégradation des sols au Burkina Faso et en particulier dans le Centre-Nord, est un des facteurs critiques pour la sécurité alimentaire et l'amorce d'une croissance agricole.

Cette étude permet de faire le point de la situation sur la compréhension du phénomène par les paysans et plus particulièrement de comparer les pratiques paysannes aux potentiels des différentes unités géomorphologiques dans le Centre-Nord du Burkina Faso.

\section{MATERIEL ET METHODES}

Cadre de l'étude

La région du Centre-Nord fait partie intégrante de la pénéplaine centrale du Burkina Faso. Elle couvre une superficie totale de $19508 \mathrm{~km}^{2}$. Située entre le $12^{\circ} 40^{\prime} \mathrm{N}$ et $14^{\circ} \mathrm{N}$, elle regroupe les trois provinces du
Bam, du Samnatenga et du Namentenga. La province du Bam, avec pour chef lieu Kongoussi, intègre les sous-bassins de Minima et de Loulouka, ce dernier faisant l'objet de la présente étude (figure 1).

Ce sous-bassin fait partie du domaine climatique sahélo-soudanien, caractérisé par une longue saison sèche (novembre à mai) et une courte saison pluvieuse (juin à octobre). La classification des climats, selon les précipitations, faite par Fontès et Guinko (1995), place celui du site d'étude dans le type dit de transition sahélo-soudanien avec une pluviosité comprise entre 600 et $700 \mathrm{~mm}$.

La couverture géologique du site de l'étude comprend principalement des formations du Birimien (Hottin et Ouédraogo, 1992). Ces formations sont composées de roches volcano-sédimentaires (tufs, laves, sédiments associés, métavolcanites neutres à basiques).

Le relief se présente sous la forme de collines à cuirasses sommitales affleurantes et aux versants à pentes fortes. Les collines birimiennes sont essentiellement composées de schistes, de roches vertes, de granites et de formations superficielles. Elles constituent l'ossature du modelé de la zone d'étude.

Le paysage géomorphologique s'organise depuis les reliefs résiduels qui conservent par endroit des lambeaux de cuirasse ferro-alumineuse, aux bas-fonds. Entre les deux, se succèdent dépressions, glacis cuirassés et glacis non-cuirassés. Les dépressions se sont développées à la périphérie des reliefs élevés, à la faveur de la dynamique érosive dans la zone de raccordement entre versant à pente forte $\left(>30^{\circ}\right)$ et glacis cuirassé faiblement incliné (1 à $\left.5^{\circ}\right)$. Ce dernier, sapé en amont et disséqué en aval par les eaux de ruissellement, parfois morcelé par des entonnoirs de percée, se transforme en lambeaux. Cela permet à un autre glacis plus ou moins long, fonctionnel cette fois, de le séparer du bas-fond ou des lits majeur et mineur du cours d'eau, véritable collecteur des eaux de pluie (Da, 2004; Legoupil, 1995 ; figure 2).

Sur ces unités géomorphologiques, les roches plus ou moins altérées et les matériaux colluvionnaires favorisent le développement de différents types de sols: regosols sur cuirasse, lixisols et cambisols sur glacis non cuirassés, sur interfluves de roches vertes et 
dans les dépressions fluvisols et gleysols dans les bas-fonds et les plaines. Les vertisols se localisent par endroits dans les dépressions, les plaines alluviales alimentées en matériaux provenant de roches basiques, les alvéoles et les bassins de décantation, les collines de roches volcaniques basiques du Birimien.

La population de la zone est estimée à 24.212 habitants (INSD, 2007). La densité moyenne est de 37 habitants au $\mathrm{km}^{2}$ contre une moyenne nationale de 29. Le taux de croissance est estimé à $1,72 \%$ contre $2,7 \%$ pour la moyenne nationale. Seulement $4 \%$ de cette population est alphabétisée et 14,3\% scolarisée.

La comparaison des données statistiques de l'INSD de 1996 (18.607 habitants) et 2007 permet de dire que la population s'est accrue de 5605 âmes en 10 ans, soit 30,12\%. Il est alors facile de comprendre les conséquences d'une telle explosion démographique sur les ressources naturelles, surtout lorsque des mesures d'exploitation rationnelles du milieu ne sont pas observées.

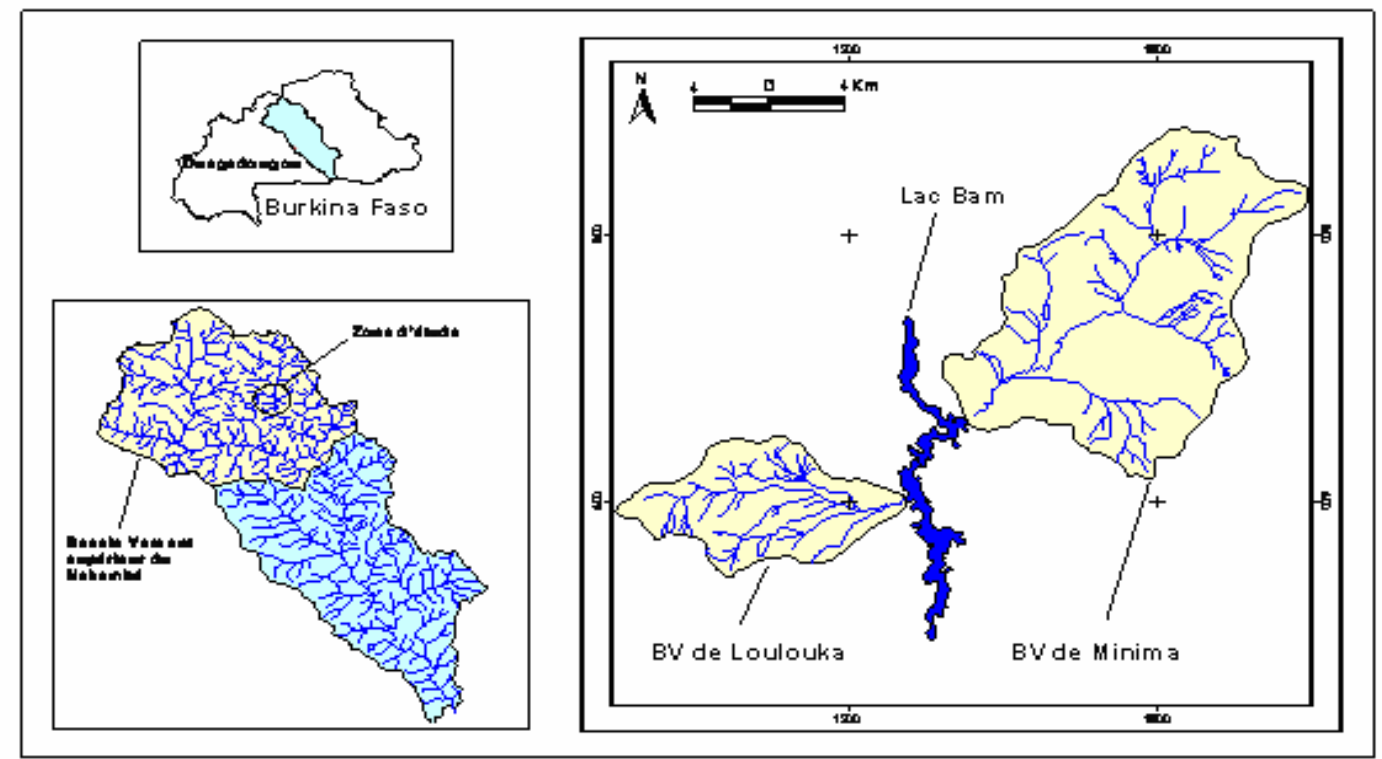

Figure 1 : Situation géographique de la zone d'étude.

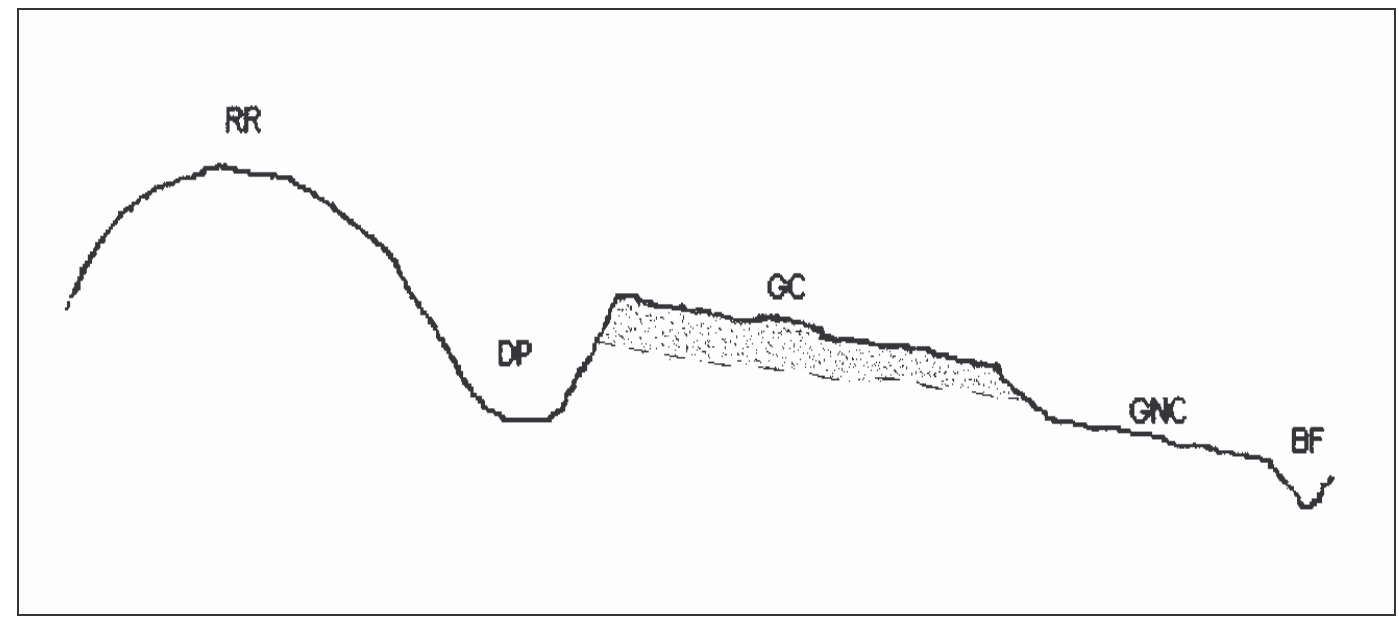

Figure 2 : Morphologie typique de la zone d'étude. $\mathrm{RR}=$ Relief résiduel ; DP = Dépression périphérique ; $\mathrm{GC}=$ Glacis cuirassé $; \quad \mathrm{GNC}=$ Glacis non cuirassé $; \mathrm{BF}=$ Bas-fond. 


\section{Méthodologie}

$\mathrm{Au}$ vu des objectifs fixés, la démarche adoptée vise à faire le point sur le potentiel agronomique des sols et sa perception par les populations locales.

\section{Potentiel agronomique des sols}

Des échantillons de sols ont été prélevés à la tarière, à trois profondeurs d'horizons : de 0 à $20 \mathrm{~cm}$, de 20 à $40 \mathrm{~cm}$ et de 40 à $60 \mathrm{~cm}$. Ces échantillons ont fait l'objet d'analyses granulométrique et chimique au Bureau National des Sols (BUNASOLS) du Burkina Faso. L'analyse granulométrique permet d'estimer les dépôts dans les basfonds, zone de concentration des eaux de ruissellement. L'analyse chimique a concerné les paramètres suivants consignés dans le tableau 1 et qui indique la méthode d'analyse pour chaque paramètre.

Ces analyses ont eu pour but d'estimer le niveau de fertilité des sols des unités géomorphologiques, corrélativement avec la perception des agriculteurs qui les exploitent.

Le critère de classification de niveau de fertilité est basé sur celui du BUNASOLS (1990) où les paramètres considérés sont la matière organique, la somme des bases échangeables et le $\mathrm{pH}$ eau; un chiffre d'appréciation (cotation de 1 à 5) constitue une norme d'interprétation pour chacun d'eux. Ainsi les intervalles des teneurs et les normes sont basés sur la gamme des pourcentages de chaque paramètre telle que rencontrée dans les sols du Burkina Faso, tout en tenant compte des normes internationales. Le tableau 2 donne ainsi les cotations pour chaque paramètre et la somme de cotations des trois cités. Cette somme permet ainsi de déduire la classe de fertilité pour un sol donné.

Perception du potentiel de fertilité par les populations locales

L'approche ethno-pédologique aidera à déterminer la connaissance que les populations locales ont sur le niveau de fertilité des sols qu'elles exploitent et ce, au travers de leurs pratiques agricoles. Plusieurs outils de recueil de l'information ont été mobilisés pour cet aspect d'analyse du milieu. Cette approche est basée sur les enquêtes avec des questionnaires individuels et des guides d'entretien semi-directifs.

Tableau 1: Paramètres chimiques et méthodes d'analyse.

\begin{tabular}{lll}
\hline Eléments chimiques & Méthodes d'analyse & Sources \\
\hline Matière Organique et carbone & $\begin{array}{l}\text { Walkey et Black } \\
\text { Azote total }\end{array}$ & Houba et al., 1995. \\
$\begin{array}{l}\text { Calcium total et magnesium total } \\
\text { Potassium total }\end{array}$ & $\begin{array}{l}\text { Spectrophotométrie } \\
\text { Extrait par acide } \\
\text { chlorhydrique-oxalique }\end{array}$ & \\
\cline { 1 - 2 } Capacité d'Echange Cationique $(\mathrm{CEC})$ & Argent thio-urée & \\
Potassium échangeable & & \\
Bases échangeables $\left(\mathrm{Ca}^{++}, \mathrm{Mg}^{++}, \mathrm{Ka}^{+}, \mathrm{Na}^{+}\right)$ & & Dewis et Freitas, 1984. \\
\hline Particules pour analyse granulométrique & - hydrométrique & \\
-3 fractions & - Pipette Robinson & \\
-5 fractions & & \\
\hline
\end{tabular}

Tableau 2: Classe de fertilité des sols au Burkina Faso.

\begin{tabular}{lllllll}
\hline $\begin{array}{l}\text { Classe (interprétation) pour } \\
\text { chaque paramètre }\end{array}$ & Très bas & Bas & Moyen & élevé & Très élevé \\
\hline Matière organique & $\%$ & $<0,5$ & $0,5-1$ & $1-2$ & $2-3$ & $>3$ \\
& Cotation & 1 & 2 & 3 & 4 & 5 \\
\hline Somme des bases & méq/100g & $<1$ & $1-6$ & $6-11$ & $11-16$ & $>16$ \\
échangeables & Cotation & 1 & 2 & 3 & 4 & 5 \\
\hline pH eau & Valeurs & $<4,5$ & $4,6-5$ & $5,1-5,5$ & $5,6-6$ & $6,1-7,3$ \\
& & $>9$ & $8,5-9$ & $7,9-8,4$ & $7,4-7,8$ & \\
\cline { 2 - 7 } & Cotation & 1 & 2 & 3 & 4 & 5 \\
\hline \multicolumn{2}{l}{ Somme des cotations : } & $<4,4$ & 4,5 à 7,5 & 7,6 à 10,5 & 10,6 à 13,5 & $>13,6$ \\
\hline \multicolumn{2}{l}{ Classe de fertilité des sols } & Très bas & Bas & Moyen & élevé & Très élevé \\
\hline \multicolumn{2}{l}{ Source: BunAsols, 1990. } & & & & &
\end{tabular}


Sur l'ensemble du bassin versant de Loulouka, 50 personnes ont été interrogées. La tranche d'âge des enquêtés est comprise entre 20 et 50 ans.

\section{RESULTATS}

Les sols opposent une résistance à l'érosion hydrique. Au regard de l'agressivité climatique du couvert végétal et des pratiques agricoles, la résistance des sols à l'érosion hydrique est liée à leur texture (surtout à leur teneur en argile) et à la quantité de matière organique (Roose, 1994). Toute dégradation qui en résulte du fait d'une forte érodibilité du sol peut entraîner, par la suite, un transport d'éléments fins, notamment les bases échangeables. Les résultats d'analyse de sols, consignés dans le tableau 1 , permettent ainsi d'évaluer les conséquences de l'érosion hydrique sur la valeur agronomique des sols du site étudié.

\section{Texture et Matière organique}

Le tableau 1 montre que les sols des dépressions sont très pauvres en argile (moins de $2 \%$ sur l'ensemble des horizons) alors que ceux des bas-fonds et des longs glacis en sont très riches (en moyenne 15\%). Les lithosols sur cuirasses, aussi bien de sommet que de glacis, malgré leur compacité qui limite leur exploitation agricole, sont aussi riches en argile particulièrement en surface. A contrario, les dépressions sont très riches en sable (en moyenne $84 \%$ ) alors que les sols de toutes les unités géomorphologiques restent dans des proportions comparables à ceux des sols dits francs.

Les limons, très faibles dans les dépressions en général, sont par contre très présents dans les 20 premiers centimètres des glacis. Ce qui explique l'encroûtement de ces derniers (Ambouta, 1994), qui les rend de ce fait imperméables (Casenave et Valentin, 1989). Les études de Zombré (2006a) montrent que ces glacis, qui occupaient $0,2 \%$ des superficies de la région en 1955, sont passés à 1,8\%. Ceci dénote une importante extension. La mise en valeur agricole de ces unités ne se fait actuellement que par les techniques de restauration des sols dégradés telles le «zaï » et les demi-lunes (Kambou et Zougmoré, 1996; Mando et al., 2001 ; Zombré, 2006b).

La matière organique, quasiment inexistante sur l'ensemble des unités, indique la nécessité absolue d'apporter de la fumure organique (et non uniquement des engrais minéraux) sur ces sols, afin de maintenir leur fertilité. La gestion de la matière organique est d'ailleurs au cœur de la problématique sur la fertilité des sols au Burkina Faso (Sédogo et al., 1989, 1995 ; Lompo et al., 1994, 1995, 1996 ; Yacouba et al., 2001).

\section{Fertilité chimique}

Le tableau 3 donne les résultats d'analyses chimiques sur les quatre unités géomorphologiques de la zone d'étude. Ces résultats permettent de classer ces unités selon leur niveau de fertilité. La classification est basée sur les normes d'interprétation développées dans le tableau 2.

Ainsi, sur un même transect, il a été regroupé les unités géomorphologiques semblables puis, pour l'ensemble des transects celles au niveau du bassin de Loulouka, ceci pour faire une analyse de la fertilité des sols de chaque unité du sous-bassin.

Pour pouvoir déterminer la disponibilité en éléments nutritifs (niveau de fertilité) et la capacité de rétention de ces éléments des sites qui ont fait l'objet d'échantillonnage, nous avons procédé à la détermination des classes dont le résultat est consigné dans le tableau 4.

De l'analyse du tableau 4, il ressort que :

- les bas-fonds sont les unités les plus propices à l'agriculture, du fait de leur richesse en bases échangeables et en matières organiques par rapport aux autres unités.

- Ils sont suivis par les glacis d'épandage qui sont moyennement riches sur l'ensemble de leur profil ; la différence d'avec les bas-fonds réside dans l'accumulation des éléments minéraux dans les couches inférieures de ces sols, notamment les horizons B1 et B2. ces éléments minéraux qui entrent ensuite dans la formation du sol avec la décomposition de la roche mère, sont des atouts majeurs en potentialité agronomique de ces derniers.

Les dépressions, très sableuses, sont très pauvres en éléments fertilisants et particulièrement en bases échangeables. Cette situation viendrait du fait que les sols de cette unité géomorphologique sont soumis à un lessivage important qui limite leur fertilité. Ces résultats corroborent celles des études de Gerhard (1994). 
Tableau 3: Analyses physicochimiques des sols des principales unités géomorphologiques.

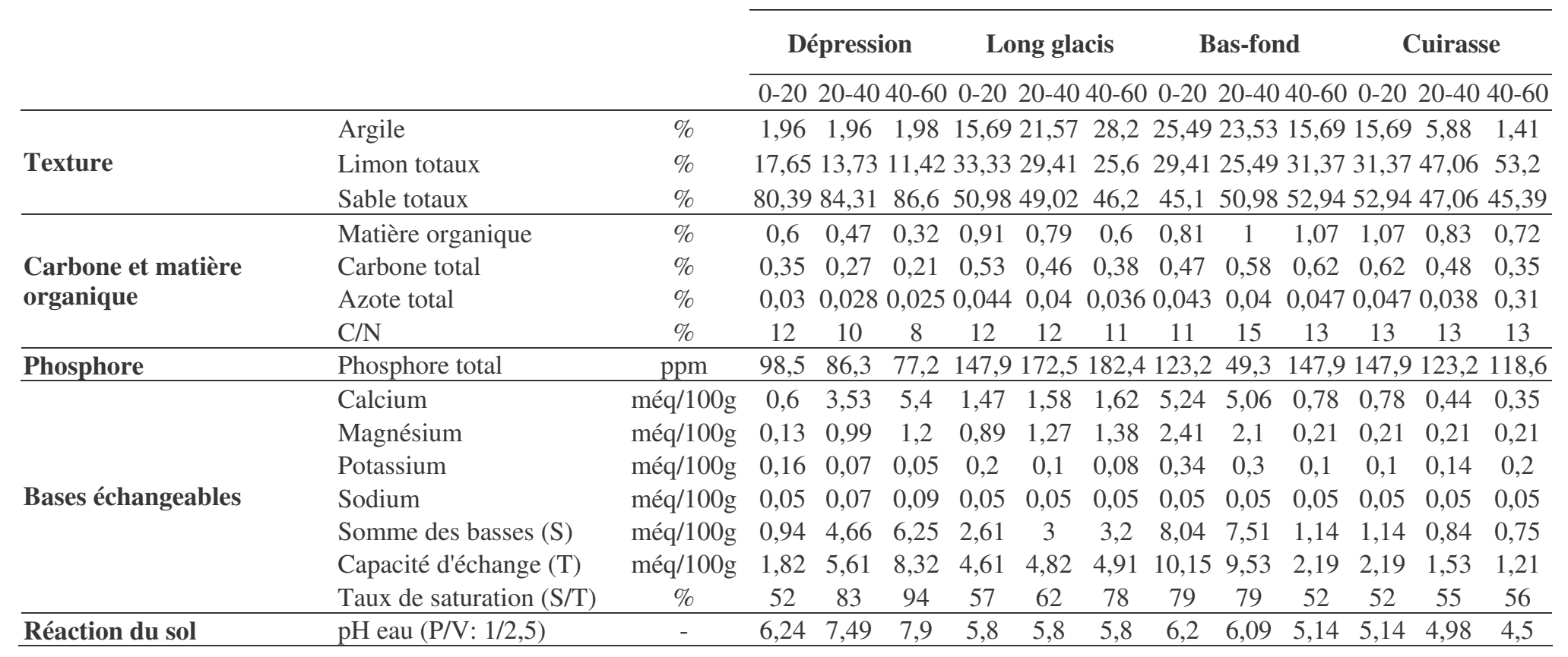


Tableau 4: Classes de fertilité des sols des différentes unités géomorphologiques du sous-bassin de Loulouka.

Profondeur Matière Somme des

(cm) organique bases pH eau Somme des Classe de (\%) (méq/100g) cotations fertilité

\begin{tabular}{|c|c|c|c|c|c|c|}
\hline \multirow{6}{*}{ Dépression } & $0-20$ & 0,6 & 0,94 & 6,24 & & \\
\hline & cotation & 2 & 1 & 5 & 8 & Moyenne \\
\hline & $20-40$ & 0,47 & 4,66 & 7,49 & & \\
\hline & cotation & 1 & 2 & 4 & 7 & Basse \\
\hline & $40-60$ & 0,32 & 6,25 & 7,9 & & \\
\hline & cotation & 1 & 3 & 3 & 7 & Basse \\
\hline \multirow{6}{*}{$\begin{array}{l}\text { Long glacis } \\
\text { (glacis cuirassé } \\
\text { et glacis non } \\
\text { cuirassé) }\end{array}$} & $0-20$ & 0,91 & 2,61 & 5,8 & & \\
\hline & cotation & 2 & 2 & 4 & 8 & Moyenne \\
\hline & $20-40$ & 0,79 & 3 & 5,8 & & \\
\hline & cotation & 2 & 2 & 4 & 8 & Moyenne \\
\hline & $40-60$ & 0,6 & 3,2 & 5,8 & & \\
\hline & cotation & 2 & 2 & 4 & 8 & Moyenne \\
\hline \multirow{6}{*}{ Bas-fond } & $0-20$ & 0,81 & 8,04 & 6,2 & & \\
\hline & cotation & 2 & 3 & 5 & 10 & Moyenne \\
\hline & $20-40$ & 1 & 7,51 & 6,09 & & \\
\hline & cotation & 3 & 3 & 5 & 11 & Elevée \\
\hline & $40-60$ & 1,07 & 1,14 & 5,14 & & \\
\hline & cotation & 3 & 2 & 3 & 8 & Moyenne \\
\hline \multirow{6}{*}{ Cuirasse } & $0-20$ & 1,07 & 1,14 & 5,14 & & \\
\hline & cotation & 3 & 2 & 3 & 8 & Moyenne \\
\hline & $20-40$ & 0,83 & 0,84 & 4,98 & & \\
\hline & cotation & 2 & 1 & 2 & 5 & Basse \\
\hline & $40-60$ & 0,72 & 0,75 & 4,5 & & \\
\hline & cotation & 2 & 1 & 1 & 4 & Très bas \\
\hline
\end{tabular}

Appréciation des valeurs agronomiques des sols par les paysans et stratégies de mise en valeur

Les paysans Mossi de la région de Loulouka, sinon du Burkina Faso donnent aux sols des noms en rapport d'une part avec les caractéristiques et partant les valeurs agronomiques, et d'autre part avec la topographie ou les unités géomorphologiques.
Baongo désigne le sol de bas-fond. En ce sens, le mot sous-entend présence d'eau et fait appel au caractère hydromorphe du sol. Sur ce sol le plus souvent limoneux à argileux, quelque fois sablo-argileux, les paysans $\mathrm{y}$ cultivent mil, sésame, riz pluvial, patate, calebasse et sorgho lorsque la teneur en argile est élevée. 
Bollé désigne le sol argileux des plaines, des versants et de certaines dépressions. Il est exploité en culture de sorgho et de riz pluvial lorsque les conditions d'hydromorphie le permettent. Il a un caractère vertique.

Bisri ou bin-hiri regroupe les sols sableux se développant sur les glacis actuels, fonctionnels sur lesquels sont cultivés : mil, arachide, haricot, pois de terre, ...

Zengdga est le terme utilisé pour désigner les sols gravillonnaires des glacis cuirassés ou non, exploités en mil, quelques rares fois en sorgho.

Kougouri caractérise les affleurements rocheux, les sommets et buttes cuirassés, seulement exploités en sorgho lorsque s'y sont développés des regosols.

Par le choix des espèces à cultiver sur ces différents types de sols, les paysans prouvent qu'ils en ont une bonne connaissance et une maîtrise de leurs aptitudes agronomiques. Leur activité principale est donc l'agriculture. Toutefois l'élevage et le commerce constituent des activités secondaires. Les bas-fonds sont les zones les plus prisées pour l'agriculture et pour lesquels, on a le plus de conflits. L'agriculture se pratique aussi sur les glacis d'épandage, les dépressions moins fertiles et les terres marginales des collines et des cuirasses à cause de la faible extension des bas-fonds. Le matériel utilisé à cette fin est la daba.

Dans l'ensemble, les paysans sont conscients de la dégradation et cela depuis une vingtaine d'années. La principale cause de dégradation évoquée est la sécheresse avec pour corollaire l'action éolienne. Viennent ensuite la coupe abusive de bois, les pratiques agricoles et pastorales néfastes. Pratiquement tous les paysans connaissent les techniques de conservation des eaux et des sols (digues, diguettes et cordons pierreux par exemple), à cause des nombreuses interventions des projets de CES/AGF dans la zone. Cependant $30 \%$ des enquêtés ne les utilisent pas par manque de moyens.

\section{DISCUSSION}

L'objectif de l'étude était de faire le point sur la relation entre l'organisation des unités géomorphologiques et les sols d'une part, et d'autre part entre les unités morpho pédologiques et leur perception par les populations qui les exploitent, dans un milieu qui se dégrade de plus en plus, ceci en vue de cerner toute l'importance du modelé dans le drainage hydrique et dans la fertilité des sols. L'approche qui a été adoptée a permis de caractériser les sols suivant leur disposition géomorphologique, d'analyser leurs qualités physicochimiques, de dégager leur potentiel agronomique et de comparer les pratiques paysannes sur les différentes unités géomorphologiques. $\mathrm{Ce}$ potentiel agronomique a été établi sur la base d'analyses de sols au laboratoire. Ces analyses ont relevé une bonne concordance entre le potentiel agricole de ces unités et l'exploitation qu'en font les paysans. Ainsi les regosols, qu'ils soient sur les sommets ou sur glacis, sont exploités après récupération par les techniques de zaï ou de demi-lunes. En effet, la présence d'argiles en surface sur ces unités les prédispose à une bonne teneur en bases échangeables, comme l'ont confirmé les analyses physicochimiques. Ces regosols sont presque aussi riches que les sols de basfonds; mais leurs surfaces endurées, très difficiles à travailler et avec une faible capacité d'infiltration ne facilite pas leur exploitation par de simples techniques de labours traditionnels (Casenave et Valentin, 1989). Le mil et le sorgho, sont généralement cultivés dans les poquets de «zaï» et de demi-lunes qui constituent des zones de concentration des eaux de ruissellement sur ces unités aménagées.

Les bas-fonds, jadis considérés comme des terres incultes sur le plan agricole, réservées de ce fait aux pâturages et dans certains cas aux lieux de cultes, sont aujourd'hui les terres agricoles convoitées par les paysans (Dacosta et al., 2002). Les analyses physicochimiques, réalisées sur les sols de cette unité, ont montré une bonne teneur en éléments chimiques, notamment en bases échangeables et en matières organiques. Leur potentiel agricole reste intact malgré leur exploitation par des cultures exigeantes en éléments fertilisants telles que le riz.

Les dépressions, de par leur emplacement en tant que zones de transition entre les reliefs résiduels et les glacis dans le paysage, sont riches en argiles ou en sables. Leur exploitation dépend de la dominance des ces deux éléments granulométriques et la tendance pluviométrique de la saison en cours. 
Les spéculations courantes sont souvent mises en association pour réduire les risques liés à l'irrégularité des pluies.

Même si les paysans sont conscients de la nécessité de mise en jachère dont l'effet sur la régénération ligneuse et la fertilité des sols est bien connu (Yaméogo et al., 2004), la pression foncière due à la densité de population de la région rend impossible sa mise en pratique.

\section{Conclusion}

La dégradation des sols dans le Centre Nord du Burkina et plus particulièrement dans le sous bassin de Loulouka devient préoccupante. Cette dégradation bien visible par une présence de croûte imperméable à l'eau à la surface des lithosols se traduit par l'apparition des zones nues de glacis difficiles à exploiter. Les paysans assistent, impuissants, à une baisse continue des rendements des cultures qu'ils constatent par eux-mêmes ; ce qui les fragilise davantage.

Contraints à vivre cette situation, ces paysans arrivent à s'y adapter. Ainsi, conscients de la dégradation continue de leur milieu les paysans de cette région tentent d'introduire des approches pragmatiques pour son exploitation. C'est le cas notamment des petits aménagements appelés «zaï » tendant à concentrer les eaux de pluies aux pieds des plantes cultivées pour compenser le déficit et la variabilité pluviométrique. A ces petits aménagements s'ajoute une stratégie par laquelle ces paysans exploitent les unités en fonction de leur niveau fertilité naturel : les cultures les plus exigeantes sur les unités géomorphologiques de bonne fertilité telles que les bas-fonds par exemple.

Cependant il est à souligner un manque visible de plan efficace et durable de restauration du milieu. En effet, on ne note pas une politique de fertilisation et d'amendement des sols des différentes unités morphologiques qui garantirait une stabilisation de la production. Dans cette zone, aucune action de plantation d'arbres n'est visible et la pression foncière n'autorise pas la jachère. Les seules pratiques observées se localisent au niveau des regosols sur croûtes de battance où les paysans font des efforts pour confectionner de «zaï » et de demi-lunes avec apport des quelques poignées de fumier, mais aussi au niveau des pentes où, pour stabiliser les sols contre l'érosion hydrique, les paysans utilisent les cordons pierreux ou les bandes enherbées.

Les aménagements visant d'une part la lutte contre l'érosion mécanique et d'autre part la restauration de la fertilité des sols du sous-bassin de Loulouka restent encore faibles. C'est la preuve que même si la population perçoit la dégradation des terres, des efforts devront être faits pour accélérer l'extension des pratiques de conservation des eaux et des sols.

\section{BIBLIOGRAPHIE}

Ambouta JMK. 1994. Etude des facteurs de formations d'une croûte d'érosion et de ses relations aux propriétés internes d'un sol sableux fin au sahel. Thèse de PhD. Département des sols, Faculté des sciences de l'Agriculture et de l'Alimentation, Université Laval, SainteFoy, Québec, p. 97.

BUNASOLS. 1990. Manuel pour l'évaluation des terres. Documentations techniques $\mathrm{n}^{\circ} 6$, Ouagadougou, p. 181.

Casenave A, Valentin C. 1989. Les Etats de Surface de la Zone Sahélienne. Influence sur l'Infiltration. ORSTOM: Paris; 229.

Da DEC. 2004. Evolution des milieux au Sahel central: les marges subtropicales du Burkina Faso. Dossier HDR, URCA, France, 3 vol., p. 617.

Dacosta H, Mendy A, Akpo E, Malou R, Dionne F. 2002. In Proceeding de la $5^{\text {ème }}$ Conférence Interrégionale sur l'Environnement et l'Eau - Envirowater 2002, pp.610-615.

Dewis J, Freitas F. 1984. Méthodes d'analyse physique et chimique des sols et des eaux. Bulletin Pédologique de la FAO, 10: 275.

Fontès J, Guinko S. 1995. Carte de la végétation et de l'occupation du sol du Burkina + Notice explicative. Projet Campus, U.O., p. 66.

Gerhard R. 1994. Improvement of sandy soils by clay-substrate application. Applied Clay Science, 9: 107-120.

Hottin G, Ouédraogo F. 1992. Carte géologique du Burkina Faso. Echelle 1 : 1000000 + notice explicative. 2ème édition. Direction de la géologie et des mines, Ouagadougou, p. 58.

Houba VJG, van der Lee JJ, Novozamsky I. 1995. Soil Analysis Procedures. Other 
Procedures (Soil and Plant Analysis, part 5B) (6th edn). Department of Soil Science and Plant Nutrition, Wageningen Agricultural University; 138.

INSD. 1997. Recensement général de la population et de l'habitat du Burkina Faso du 10 au 20 décembre 1996. Ministère de l'Economie et des Finances, Edition de mars.

INSD. 2007. http://www.insd.bf/, 24 Décembre 2007.

Kambou N, Zougmoré R. 1996. Evolution des états de surface d'un «zippelé » soumis à différentes techniques de restauration des sols (Yilou, Burkina Faso). Bull. Réseau Erosion, 16: 19-32.

Legoupil JC, Lidon B. 1995. La mise en valeur des bas-fonds en Afrique subsaharienne : un enjeu important lié à la connaissance et à la maîtrise de leurs régimes hydriques. In Actes du $3^{\text {ème }}$ atelier annuel du consortium bas-fonds. ADRAO-Bouaké, Côte d'Ivoire, 23-24 mars $1995 ; 71-81$.

Lompo F, Sedogo PM, Assa A. 1994. Effet à long terme des phosphates naturels de Kodjari (Burkina Faso) sur la production de sorgho. Bilans minéraux. Rev. Res. APAMA, 6: 163-178.

Lompo F, Sédogo PM, Hien V. 1995. Impact agronomique du phosphate et de la dolomie du Burkina Faso. Etude diverses sur les engrais. Rev. Res. APAMA, 12: 60-72.

Mando A, Zougmoré R, Zombré NP, Hien V. 2001. Réhabilitation des sols dégradés dans les zones semi-arides de l'Afrique subsahélienne. In La Jachère en Afrique Tropicale (Vol. II), Foret C, Pontanier R (eds). John Libbey: Paris; 311-339.
PNUE. 2002. L'avenir de l'Environnement Mondial 3. GEO-3; p. 480.

Roose E. 1994. Introduction à la gestion conservatoire de l'eau, de la biomasse et de la fertilité des sols (CGES). Bull. Pédol. FAO, 70: 240.

Sedogo MP, Lompo F, Boubié VB. 1989. Gestion de la fertilité des sols en milieu tropical. Rev. Res. APAMA, 1: 199-207.

Sedogo MP, Lompo F, Hien V, Assa A. 1995. Etude des modes de gestion de la fertilité sur les polysaccharides des sols ferrugineux lessivés du Plateau Central du Burkina Faso. Sc. Tech., 21(4): 94-95.

Yacouba H, Morel M, Hounto T. 2001. Valorisation par compostage de la biomasse de Pistia stratiotes issue de la station de lagunage de l'E.I.E.R. Revue Sud Sciences et Technologie, 7: 40-47.

Yaméogo G, Yelemou B, Traoré D. 2005. Pratique et perception paysanne dans la création de parc agroforestier dans le terroir de Vipalogo. http://popups.ulg.ac. be/Base/document.php?id=1404.

Yonkeu S, Yacouba H, Da DEC, Pare T, Kiniffo GF. 2003. Etude de la dégradation des paysages du bassin supérieur du fleuve Nakambé au Burkina. Rapport de synthèse. Groupe des Ecoles EIER-ETSHER \& EPEFL, oct. 2002-oct. 2003, p. 78.

Zombré NP. 2006a. Evolution de l'occupation des terres et localisation des sols dans le Centre-Nord du Burkina. Télédétection, 5(4): 285-297.

Zombré NP. 2006b. Variation de l'activité biologique dans les zippella (sols nus) en zone subsahélienne du Burkina Faso et impact de la technique du Zaï (techniques des poquets). Biotechnol. Agron. Soc. Environ., 10(2): 139-148. 